\title{
ESTABILIDADE FENOTÍPICA DE NOVAS VARIEDADES DE CANA-DE-AÇÚCAR PARA O ESTADO DE SÃO PAULO'
}

\author{
ARNALDO JOSÉ RAIZER ${ }^{2}$ e ROLAND VENCOVSKY³
}

\begin{abstract}
RESUMO - Cinco novas variedades de cana-de-açúcar (Saccharum officinarum L.), recomendadas para cultivo comercial pelo Programa de Melhoramento da Copersucar em 1997, foram avaliadas quanto ao nível de produtividade, estabilidade produtiva e adaptação ambiental, mediante metodologia para estimativa da estabilidade fenotípica. Os dados foram comparados com a variedade-padrão RB72454. Observou-se alta linearidade da produção em relação à melhoria ambiental, além de ótima estabilidade e nível de resposta nas variedades SP80-185, SP80-1816 e SP80-3280. A variedade SP83-5073, precoce e de alto teor de sacarose, também foi bastante estável. A variedade SP80-3480 apresentou maior dispersão dos dados, porém com alta produtividade. A variedade SP80-185 passa a ser indicada como padrão de estabilidade em futuros ensaios, sendo estável nos piores ambientes e responsiva nos melhores.
\end{abstract}

Termos para indexação: Saccharum officinarum, melhoramento vegetal, clones, adaptação ambiental.

\section{PHENOTYPIC STABILITY OF NEW SUGARCANE VARIETIES IN SÃO PAULO STATE}

\begin{abstract}
Five sugarcane (Saccharum officinarum L.) varieties recently released by the Copersucar Breeding Program were evaluated for yield, stability and environmental adaptability, using a methodology for phenotypic stability estimation. New varieties were compared with the check RB72454. Yield was highly related with environmental improvement. Varieties SP80-185, SP80-1816 and SP80-3280 were the most stable ones, showing high productivity on the best environments. SP83-5073, a variety with high sugar content and earliness, was also very stable, whereas, SP80-3480 showed higher scattering, but with high yield. Based on the parameters estimated the variety SP80-185 should be indicated as check for future trials, since it was the most adapted to poorer environments and the most responsive on better ones.
\end{abstract}

Index terms: Saccharum officinarum, plant breeding, clone, environmental adaptability.

\section{INTRODUÇÃO}

Nos programas de melhoramento de cana-deaçúcar grande número de clones são avaliados todos os anos, em ensaios realizados em diferentes condições ambientais, gerando dados de produtivi-

\footnotetext{
${ }^{1}$ Aceito para publicação em 23 de dezembro de 1998.

${ }^{2}$ Eng. Agr., M.Sc., Centro de Tecnologia Copersucar (CTC), Fitotecnia (CTFT-4), Faz. Sto. Antonio s/no, Caixa Postal 162 CEP 13400-970 Piracicaba, SP. E-mail: arnaldo@azul.ctc.com.br

${ }^{3}$ Eng. Agr., Dr., Prof. Titular, ESALQ/USP, Dep. de Genética, Caixa Postal 83, CEP 13400-970 Piracicaba, SP. E-mail:vencovsk@tambaqui.esalq.usp.br
}

dade com os quais procura-se indicar novas variedades para cada região produtora. O objetivo é a melhoria da produtividade agrícola e das características industriais das variedades, que devem ser mais bem adaptadas aos diferentes locais, tipos de solos e técnicas de cultivo, além de apresentarem maior resistência a pragas e doenças.

O estudo da estabilidade fenotípica permite sintetizar o enorme volume de informações obtido, caracterizando a capacidade produtiva, a adaptação às variações ambientais e a estabilidade de novas variedades. Diversos autores estudaram a interação entre genótipos e ambientes, os conceitos e índices de estabilidade, além de sugerirem alguns métodos para estimar a estabilidade fenotípica em plantas. 
Finlay \& Wilkinson (1963) estudaram a estabilidade em cultivares de cevada, considerando cada local ou ano como um ambiente. Os autores estabeleceram índices ambientais e determinaram os coeficientes de regressão linear $\left(\hat{\beta}_{\mathrm{i}}\right)$ entre a média da cultivar em cada ambiente e os índices ambientais correspondentes. Para eles, uma cultivar é considerada estável quando possuir $\hat{\beta}_{\mathrm{li}}=1$ (ns) e produtividade média superior ( $\hat{\beta}_{0 \mathrm{i}}$ elevada).

O método da regressão foi também utilizado por Eberhart \& Russel (1966), em milho, que introduziram um terceiro parâmetro para caracterizar as cultivares: a variância dos desvios da linearidade $\left(\hat{\sigma}_{\mathrm{di}}^{2}\right)$. A variedade ideal para os autores seria aquela que apresentasse produtividade média superior ( $\hat{\beta}_{0 \mathrm{i}}$ elevada), adaptabilidade geral ou ampla $\left(\hat{\beta}_{\mathrm{li}}=1\right)$ e previsibilidade ou estabilidade alta $\left(\hat{\sigma}_{\mathrm{di}}^{2}=0\right)$.

Em 1978, Verma et al. propuseram uma modificação nos métodos anteriores, dividindo os diversos ambientes de avaliação em dois subgrupos, que representariam os ambientes desfavoráveis e favoráveis, de acordo com o desvio em relação à média geral dos locais.

Kang \& Miller (1984) relataram que interações de genótipos por locais influenciaram o comportamento de variedades de cana-de-açúcar, assim como as estratégias de melhoramento da cultura. Os autores compararam algumas metodologias para estimar a estabilidade fenotípica, observando relação entre alguns parâmetros.

Peixoto et al. (1986) compararam vários métodos de regressão, avaliando com detalhe seis variedades de cana-de-açúcar largamente cultivadas no Estado de São Paulo. Os autores concluíram que o método bissegmentado fornecia informações mais completas, facilitando a alocação dessas variedades nos diversos ambientes estudados.

Segundo Vencovsky \& Barriga (1992), os modelos bissegmentados devem ser preferidos em relação aos modelos lineares simples, sempre que o número de ambientes avaliados for elevado, algo a partir de oito locais de experimentação.

Utilizando dados obtidos em 16 locais, Braga Júnior (1994b) caracterizou o comportamento relativo de clones SP (Copersucar) por meio do método de regressão bissegmentada. Em Alagoas, Calheiros \&
Barbosa (1996) avaliaram diversas variedades RB (antigo Planalsucar) em diferentes cortes e épocas de colheita, utilizando também a metodologia bissegmentada. Dessa forma, os diferentes genótipos puderam ser classificados quanto à produtividade $\mathrm{e}$ ao nível de resposta aos ambientes.

Neste trabalho procurou-se avaliar a estabilidade fenotípica de cinco novas variedades, integrantes da sexta geração de variedades SP (Copersucar, 1997), e estudou-se o comportamento produtivo desses novos genótipos frente às variações ambientais presentes nas condições do Estado de São Paulo.

\section{MATERIAL E MÉTODOS}

Foram obtidos dados de produtividade de colmos (toneladas de cana por hectare - TCH) e teor de sacarose dos colmos (pol por cento cana - PCC). Essas variáveis geraram a produtividade de sacarose, medida em toneladas de açúcar por hectare na média estimada de cinco cortes (TAH5), segundo Braga Júnior (1994a).

Para o cálculo de TAH5 foram usados os dados de dois cortes, obtidos nos Ensaios de Caracterização com plantios em 1992, 1993 e 1994, em 22 diferentes usinas cooperadas ou locais, distribuídos por várias regiões canavieiras do Estado de São Paulo. Tais ensaios constituem a fase final de avaliação de clones do Programa de Melhoramento da Cana-de-Açúcar da Copersucar. A relação das variedades estudadas, com seus respectivos genitores, encontrase na Tabela 1 .

Os ensaios foram instalados no mês de março de cada ano, com delineamento de blocos ao acaso, com quatro repetições, e parcelas de cinco sulcos de dez metros. As parcelas foram amostradas, colhidas e pesadas em julho na cana-planta e em agosto na cana-soca. Em cinco anos de experimentação foram conduzidos 43 ensaios, num total de 14, 16 e 13 Ensaios de Caracterização, nos anos de plantio de 1992, 1993 e 1994, respectivamente (Tabela 2).

Os dados de produtividade média de açúcar (TAH5), obtidos para cada grupo de ensaio, foram transformados em índices de ambiente do local e relacionados com a produtividade das variedades. Os resultados encontram-se expressos na forma gráfica e mediante estimativa dos parâmetros de estabilidade, obtidos em regressão bissegmentada. Compararam-se as cinco novas variedades de cana-de-açúcar (Copersucar, 1997) com a variedadepadrão comercial (RB72454), utilizando-se de parâmetros para avaliar a capacidade produtiva, o nível de resposta e de adaptação ambiental desses genótipos. 
Na análise dos parâmetros de estabilidade dividiram-se os ambientes em dois grupos, de acordo com os índices ambientais, os positivos, para os ambientes favoráveis, e os negativos, para aqueles desfavoráveis à produção. $\mathrm{O}$ modelo usado segue abaixo:

$\mathrm{Y}_{\mathrm{ij}}=\beta_{0 \mathrm{i}}+\beta_{\mathrm{li}} \mathrm{I}_{\mathrm{j}}+\beta_{2 \mathrm{i}} \mathrm{T}\left(\mathrm{I}_{\mathrm{j}}\right)+\delta_{\mathrm{ij}}+\overline{\varepsilon_{\mathrm{ij}}}$

onde:

$\mathrm{T}\left(\mathrm{I}_{\mathrm{j}}\right)=0$ se $\mathrm{I}_{\mathrm{j}} \leq 0 ;$

$T\left(I_{j}\right)=I_{j}$ se $I_{j}>0$;

TABELA 1. Apresentação das novas variedades SP.

\begin{tabular}{|c|c|c|}
\hline Variedade & Fêmea $^{1}$ & Macho $^{2}$ \\
\hline \multirow{3}{*}{$\begin{array}{l}\text { Padrão } \\
\text { RB72454 }\end{array}$} & & \multirow{3}{*}{$?^{3}$} \\
\hline & CP53-76 & \\
\hline & Ensaio de Caracterização 92 & \\
\hline SP80-185 & BO17 & $?$ \\
\hline SP80-1816 & SP71-1088 & H57-5028 \\
\hline SP83-5073 & SP71-1406 & SP71-1088 \\
\hline \multirow{3}{*}{ SP80-3480 } & Ensaio de Caracterização 93 & \multirow{3}{*}{ H61-1820 } \\
\hline & Q73 & \\
\hline & Ensaio de Caracterização 94 & \\
\hline SP80-3280 & SP71-1088 & H57-5028 \\
\hline
\end{tabular}

$\beta_{0 \mathrm{i}}=$ média geral do genótipo i $(\mathrm{i}=1,2, \ldots ., \mathrm{g})$;

$\beta_{1 \mathrm{i}}=\mathrm{e} \beta_{2 \mathrm{i}}=$ resposta linear do genótipo i à variação ambiental;

$\mathrm{I}_{\mathrm{j}}=$ índice ambiental para o local $\mathrm{j}(\mathrm{j}=1,2, \ldots ., \lambda$ sendo

$I_{j}=\frac{Y_{j}}{g}-\frac{Y}{g \lambda}$

$\delta_{\mathrm{ij}}=$ desvio de regressão;

$\overline{\varepsilon_{\mathrm{ij} .}}=$ erro experimental médio.

As estimativas e derivações matemáticas, assim como ilustrações e emprego dos parâmetros gerados, podem ser observadas em Cruz \& Regazzi (1994). As novas variedades foram avaliadas pela produtividade na média dos locais de experimentação $\left(\hat{\beta}_{0 i}\right)$ e comparadas pelos coeficientes das regressões, $\hat{\beta}_{1 \mathrm{i}}$ e $\hat{\beta}_{2 \mathrm{i}}$, que foram avaliados por meio da estatística $t$. Também obtiveram-se os coeficientes de determinação das regressões $\left(\mathrm{R}^{2}\right)$.

\section{RESULTADOS E DISCUSSÃO}

Quanto à produtividade média dos ensaios, o Ensaio de Caracterização plantado em 1992 foi o mais produtivo, seguido do plantado em 1993 e, finalmente, do ensaio com plantio em 1994 (Tabela 2).

TABELA 2. Locais estudados, médias de tonelada de açúcar por hectare e índice ambiental.

\begin{tabular}{|c|c|c|c|c|c|c|c|c|}
\hline $\begin{array}{c}\text { Locais } \\
\text { Caracterização } 92 \\
\end{array}$ & $\begin{array}{l}\text { Média } \\
\text { TAH5 }\end{array}$ & $\begin{array}{l}\text { Índice } \\
\text { amb. }{ }^{1}\end{array}$ & $\begin{array}{c}\text { Locais } \\
\text { Caracterização } 93 \\
\end{array}$ & $\begin{array}{l}\text { Média } \\
\text { TAH5 } \\
\end{array}$ & $\begin{array}{l}\text { Índice } \\
\text { amb. }\end{array}$ & $\begin{array}{c}\text { Locais } \\
\text { Caracterização } 94 \\
\end{array}$ & $\begin{array}{l}\text { Média } \\
\text { TAH5 }\end{array}$ & $\begin{array}{c}\text { Índice } \\
\text { amb. }\end{array}$ \\
\hline Barra Grande & 13,1 & $-1,76$ & Barra Grande & 13,4 & 0,21 & Barra Grande & 11,4 & $-1,32$ \\
\hline Bela Vista & 15,4 & 0,50 & Catanduva & 10,6 & $-2,60$ & Catanduva & 15,0 & 2,31 \\
\hline Cresciumal & 15,6 & 0,73 & Cresciumal & 14,8 & 1,63 & Cresciumal & 12,4 & $-0,31$ \\
\hline Iracema & 16,7 & 1,85 & Da Pedra & 10,4 & $-2,74$ & Diamante & 15,2 & 2,51 \\
\hline Santa Adélia & 14,2 & $-0,69$ & Santa Adélia & 12,3 & $-0,92$ & Santa Adélia & 12,1 & $-0,65$ \\
\hline Quatá & 13,0 & $-1,90$ & Santa Luiza & 13,9 & 0,73 & Santa Luiza & 12,2 & $-0,51$ \\
\hline Santa Maria & 13,7 & $-1,20$ & Santa Maria & 14,2 & 1,02 & Santa Maria & 11,2 & $-1,55$ \\
\hline Santa Terezinha & 14,6 & $-0,27$ & São Carlos & 12,2 & $-1,00$ & São João & 12,6 & $-0,07$ \\
\hline São João & 14,7 & $-0,22$ & São Francisco $A B$ & 14,2 & 1,06 & São Luiz AA & 9,5 & $-3,20$ \\
\hline São Luiz AA & 14,5 & $-0,37$ & São João & 14,9 & 1,69 & São Luiz SA & 12,6 & $-0,12$ \\
\hline São Luiz SA & 14,9 & 0,00 & São José da Estiva & 11,6 & $-1,54$ & São Manoel & 15,7 & 2,93 \\
\hline São Manoel & 13,6 & $-1,33$ & São José ZL & 12,3 & $-0,86$ & São Martinho & 11,7 & $-1,02$ \\
\hline São Martinho & 15,3 & 0,38 & São Luiz AA & 12,5 & $-0,68$ & Zanin & 13,7 & 1,00 \\
\hline \multirow[t]{2}{*}{ Zanin } & 19,2 & 4,27 & São Manoel & 15,5 & 2,27 & & & \\
\hline & & & Zanin & 15,1 & 1,91 & & & \\
\hline Amplitude & 6,2 & & Amplitude & 5,0 & & Amplitude & 6,1 & \\
\hline Média geral & 14,9 & & Média geral & 13,2 & & Média geral & 12,7 & \\
\hline Variância & 2,6 & & Variância & 2,5 & & Variância & 3,1 & \\
\hline C.V. $(\%)$ & 10,7 & & C.V. $(\%)$ & 11,9 & & C.V. $(\%)$ & 13,9 & \\
\hline
\end{tabular}

1 Índice ambiental, em tonelada de açúcar por hectare estimada para cinco cortes (TAH5). 
Com relação à amplitude ambiental dentro de cada ano de plantio, o Ensaio de Caracterização 93, mesmo com o maior número de locais, apresentou médias com amplitude de apenas 5,0 $\mathrm{t}$ açúcar/ha (TAH5), seguido do ensaio de 94, com 6,1 TAH5, e do ensaio de 92, com amplitude igual a 6,2 TAH5. Pela análise dos coeficientes de variação das médias dos locais, os ensaios plantados em 1994 e 1993 foram os que apresentaram maior variabilidade. A partir desses dados pode-se afirmar que os clones foram submetidos a condições ambientais bem variáveis, o que é importante para estudos mais confiáveis de estabilidade fenotípica.

Procedida cada análise de variância (ANOVA) individual, com verificação da variabilidade existente entre os genótipos avaliados, realizou-se a ANOVA conjunta para cada grupo de ensaios, observandose alta significância dos efeitos genéticos e ambientais. O comportamento diferenciado das variedades, em função dos locais de experimentação, caracterizado pela alta significância da interação genótipo x locais (Tabela 3), justifica os estudos de adaptabilidade e estabilidade fenotípica.

A produtividade de açúcar (TAH5), medida pelo parâmetro $\hat{\beta}_{0 i}$, aponta as variedades SP80-3480, SP80-1816 e SP80-3280 como as mais produtivas, sendo a primeira superior à RB72454. Essa performance favorável se deve principalmente ao melhor desempenho na cana-soca, em comparação à variedade-padrão. Todas as novas variedades SP, com exceção da SP80-3480, apresentaram baixa dispersão dos dados, com elevados coeficientes de determinação $\left(\mathrm{R}^{2}\right)$, o que indica alta confiabilidade no tipo de resposta ambiental determinado pelas regressões (Tabela 4).
Desconsiderando-se a RB72454 (Car. 94), a variedade SP80-1816 foi a mais responsiva nos piores ambientes até os intermediários $\left(\hat{\beta}_{\mathrm{li}}>1\right)$, enquanto a SP80-185 destacou-se pela alta responsividade aos ambientes mais favoráveis $\left(\hat{\beta}_{2 i}>1\right)$. Por outro lado, os baixos valores de $\hat{\beta}_{1 \mathrm{i}}$ determinados para as variedades SP80-185 e SP83-5073, embora não-significativos, demonstram que tais genótipos não são tão sensíveis à adversidade do meio, ou seja, podem ser considerados mais rústicos que os demais. Essa característica é muito importante para adaptação a ambientes menos favoráveis, como em condições de solos de menor fertilidade, por exemplo. O menor valor de $\hat{\beta}_{2 i}$ encontrado para a SP80-3480 indica que a variedade não responde à melhoria ambiental ou aplicação de tecnologia, sendo recomendada para ambientes intermediários.

Em termos gerais, a variedade SP80-185 comportou-se como o genótipo mais próximo do ideal, pois mostrou ótima resposta à melhoria ambiental, sendo estável ou tolerante nos piores ambientes. A variedade SP80-1816 obteve bons resultados nos ambientes ruins a intermediários, e foi responsiva à melhoria do ambiente. As variedades SP83-5073 e SP80-3280 apresentaram comportamento igual à média dos ambientes, com boa responsividade à melhoria ambiental.

Em vários casos os parâmetros das regressões foram significativos, demonstrando associação entre a produtividade das variedades e a capacidade produtiva do ambiente. Pela baixa dispersão apresentada por grande parte dos dados, o ajuste das equações foi muito bom, confirmando a eficiência da metodologia. A avaliação desses parâmetros e o comportamento das variedades estão demonstrados na Fig. 1.

TABELA 3. Análise de variância da característica produtividade de açúcar estimada para cinco cortes (TAH5). Análise conjunta para cada grupo de ensaios.

\begin{tabular}{|c|c|c|c|c|c|}
\hline \multicolumn{6}{|c|}{ Quadro da Anova } \\
\hline \multicolumn{2}{|c|}{ Ensaios Caracterização 92} & \multicolumn{2}{|c|}{ Ensaios Caracterização 93} & \multicolumn{2}{|c|}{ Ensaios Caracterização 94} \\
\hline Fontes de variação & F Calc. & Fontes de variação & F Calc. & Fontes de variação & F Calc. \\
\hline Blocos/locais & & Blocos/locais & & Blocos/locais & \\
\hline Genótipos & $14,05 * *$ & Genótipos & $20,24 * *$ & Genótipos & $16,73 * *$ \\
\hline Locais & $59,01 * *$ & Locais ${ }^{1}$ & $36,62 * *$ & Locais & $75,18 * *$ \\
\hline Genótipos $\mathrm{x}$ locais & $7,17 * *$ & Genótipos $\mathrm{x}$ locais & $6,60 * *$ & Genótipos $\mathrm{x}$ locais & $5,66^{* *}$ \\
\hline Erro & & Erro & & Erro & \\
\hline Média & 14,90 & Média & 13,19 & Média & 12,73 \\
\hline $\mathrm{CV}(\%)$ & 6,49 & $\mathrm{CV}(\%)$ & & $\mathrm{CV}(\%)$ & 6,57 \\
\hline Parâmetros genéticos & & Parâmetros genéticos & & Parâmetros genéticos & \\
\hline Var. genética & 1,56 & Var. genética & 1,86 & Var. genética & 1,20 \\
\hline Var. locais & 2,52 & Var. locais & 1,91 & Var. locais & 2,47 \\
\hline Var. gen.xlocais & 1,38 & Var. gen.xlocais & 1,24 & Var. gen.xlocais & 0,77 \\
\hline
\end{tabular}


TABELA 4. Parâmetros de estabilidade fenotípica e dispersão das novas variedades SP em relação à variedade-padrão RB72454, com base na característica produtividade de açúcar estimada para cinco cortes (TAH5).

\begin{tabular}{|c|c|c|c|c|c|c|c|}
\hline \multicolumn{5}{|c|}{ Parâmetros da regressão bissegmentada } & \multirow{3}{*}{$\begin{array}{l}\text { Nível de } \\
\text { dispersão }\end{array}$} & \multicolumn{2}{|c|}{ Adaptação aos ambientes } \\
\hline Variedade & & & & & & Piores & Melhores \\
\hline & $\hat{\beta}_{\mathrm{oi}}$ & $\hat{\beta}_{1 \mathrm{i}}$ & $\hat{\beta}_{2 \mathrm{i}}$ & $\mathrm{R}^{2}$ & & & \\
\hline RB72454 (Car. 92) & 16,8 & $1,32^{\mathrm{ns}}$ & $1,32^{\mathrm{ns}}$ & $73 \%$ & & & \\
\hline SP80-185 & 15,4 & $0,80^{\mathrm{ns}}$ & $1,76^{* *}$ & $91 \%$ & Muito baixo & Boa & Ótima \\
\hline SP80-1816 & 16,8 & $1,98 * *$ & $1,33^{\mathrm{ns}}$ & $81 \%$ & Baixo & Ruim & Boa \\
\hline SP83-5073 & 15,6 & $0,88^{\mathrm{ns}}$ & $1,06^{\mathrm{ns}}$ & $83 \%$ & Baixo & Boa & Média \\
\hline RB72454 (Car. 93) & 13,2 & $0,66^{*}$ & $1,96^{* *}$ & $79 \%$ & & & \\
\hline SP80-3480 & 15,0 & $1,25^{\mathrm{ns}}$ & $0,59^{\mathrm{ns}}$ & $66 \%$ & Médio & Média & Ruim \\
\hline RB72454 (Car. 94) & 15,1 & $1,91 * *$ & $0,27^{* *}$ & $73 \%$ & & & \\
\hline SP80-3280 & 14,7 & $1,19^{\mathrm{ns}}$ & $1,19^{\mathrm{ns}}$ & $79 \%$ & Baixo & Média & Média \\
\hline
\end{tabular}

ns, ${ }^{*}{ }^{* *}$ Não-significativo e significativo a $5 \%$ e $1 \%$, respectivamente, pelo teste t.
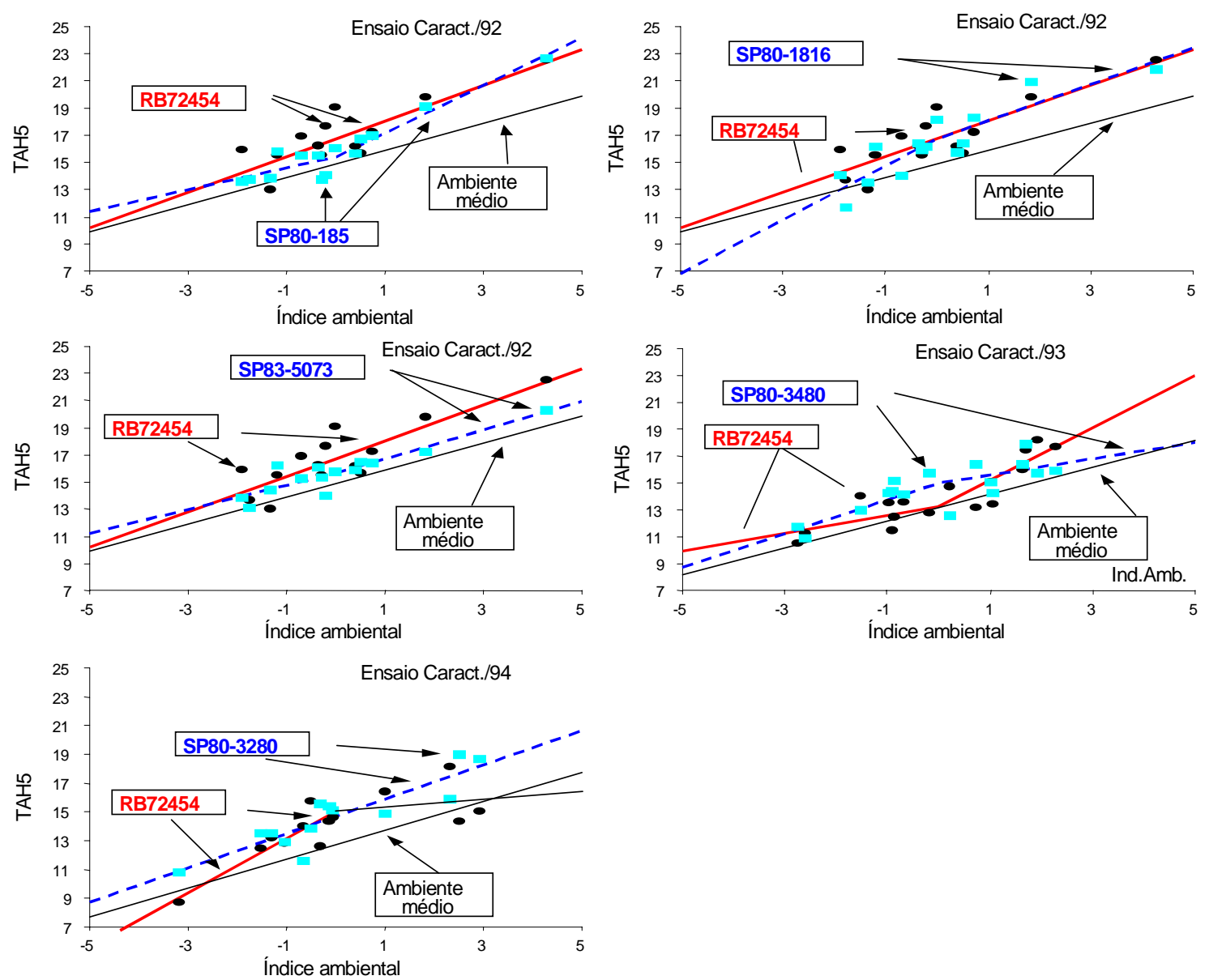

FIG. 1. Estabilidade das novas variedades SP em comparação com a variedade RB72454. Curvas de regressão bissegmenteada, com base na característica produtividade de açúcar estimada para a média de cinco cortes (TAH5). 


\section{CONCLUSÕES}

1. A produtividade de açúcar das variedades avaliadas está linearmente relacionada com a melhoria ambiental.

2. Em virtude do ótimo desempenho na soqueira, as variedades SP80-3480, SP80-1816 e SP80-3280 alcançam as maiores produtividades de açúcar, na média estimada para cinco cortes.

3. As variedades SP80-185, SP80-1816, SP83-5073 e SP80-3280 apresentam baixa dispersão de produção nos diferentes locais avaliados, com boa previsibilidade de resposta à melhoria ambiental.

4. O tipo de adaptação ambiental qualifica as variedades SP80-185 e SP80-1816 como altamente rentáveis nos melhores ambientes de produção; por sua vez, SP80-3480 e SP83-5073 mostram melhor adaptação nos ambientes desfavoráveis a intermediários.

5. Pelo seu comportamento característico, a variedade SP80-185 é mais indicada como padrão de estabilidade para futuros ensaios, ou seja, é estável nos piores ambientes e responsiva nos melhores.

\section{REFERÊNCIAS}

BRAGA JÚNIOR, R.L.C. Equação para estimativa da produtividade média de cinco cortes a partir dos dois primeiros cortes. In: SEMINÁRIO DE TECNOLOGIA AGRONÔMICA, 6.,1994, Piracicaba. Anais. Piracicaba: COPERSUCAR, 1994a. p.131-136.

BRAGA JÚNIOR, R.L.C. Interação genótipo-ambiente em novos clones de cana-de-açúcar. In: SEMINÁRIO DE TECNOLOGIA AGRONÔMICA, 6. 1994, Piracicaba. Anais. Piracicaba: COPERSUCAR, 1994b. p.119-129.
CALHEIROS, G.G.; BARBOSA, G.V.S. Análise da interação de genótipos RB de cana-de-açúcar (Saccharum spp.) com ambientes de cultivo em Alagoas. In: CONGRESSO NACIONAL DA SOCIEDADE DOS TÉCNICOS AÇUCAREIROS E ALCOOLEIROS DO BRASIL (STAB), 6., 1996, Maceió. Anais. Maceió: STAB, 1996. p.253-262.

COPERSUCAR. Sexta geração de variedades de canade-açúcar Copersucar. São Paulo, 1997. 28p. (Boletim técnico. Edição especial).

CRUZ, C.D.; REGAZZI, A.J. Modelos biométricos aplicados ao melhoramento genético. Viçosa: Universidade Federal de Viçosa, 1994. 390p.

EBERHART, S.A.; RUSSEL, W.A. Stability parameters for comparing varieties. Crop Science, v.6, p.3640, 1966

FINLAY, K.W.; WILKINSON, G.N. The analysis of adaptation in a plant breeding programme. Australian Journal of Agricultural Research v.14, p.742-754, 1963.

KANG, M.S.; MILLER, J.D. Genotype-environment interactions for cane and sugar yield and their implications in sugarcane breeding. Crop Science, v.24, p.435-440, 1984.

PEIXOTO, T.C.; SILVA, J.G.C.; BARRETO, M.N. Técnicas de análises de interação genótipo por ambiente e estabilidade de clones de cana-de-açúcar. In: SEMINÁRIO DE TECNOLOGIA AGRONÔMICA, 3., 1986, Piracicaba. Anais. Piracicaba: COPERSUCAR, 1986. p.11-21.

VENCOVSKY, R.; BARRIGA, P. Genética biométrica no fitomelhoramento. Ribeirão Preto: Sociedade Brasileira de Genética, 1992. 486p.

VERMA, M.M.; CHAHAL, G.S.; MURTY, B.R. Limitations of conventional regression analysis: a proposed modifications. Theoretical and Applied Genetics, v.53, p.89-91, 1978. 